University of Nebraska - Lincoln

DigitalCommons@University of Nebraska - Lincoln

Winter 2-6-2021

\title{
Nephrology Publications of Bibliographic Coupling and Co- authorship Network using VOS viewer: A Scientometric Profile
}

Chandran Velmurugan Dr

Librarian and Head Meenakshi Medical College Hospital and Research Institute (MMCH \& RI), Kanchipuram - 631 552, Chennai. Tamilnadu, India., murugan73@gmail.com

Ramasamy G Dr

Librarian Hindustan College of Arts \& Science, Padur, Chennai-603 103, Tamilnadu, India, g_ramasamy@ymail.com

Follow this and additional works at: https://digitalcommons.unl.edu/libphilprac

Part of the Library and Information Science Commons, and the Medicine and Health Sciences Commons

Velmurugan, Chandran Dr and G, Ramasamy Dr, "Nephrology Publications of Bibliographic Coupling and Co-authorship Network using VOS viewer: A Scientometric Profile" (2021). Library Philosophy and Practice (e-journal). 5014.

https://digitalcommons.unl.edu/libphilprac/5014 


\title{
Nephrology Publications of Bibliographic Coupling and Co-authorship Network using VOS viewer: A Scientometric Profile
}

\section{Dr. Chandran Velmurugan}

\author{
Librarian and Head \\ Meenakshi Medical College Hospital and Research Institute (MMCH \& RI), \\ Kanchipuram - 631 552, Chennai. Tamilnadu, India. \\ Email: murugan73@gmail.com \\ and \\ Dr. G. Ramasamy \\ Librarian \\ Hindustan College of Arts \& Science, Padur, \\ Chennai-603 103, Tamilnadu, India \\ Email: g_ramasamy@ymail.com
}

\begin{abstract}
The study has made an attempt to explore the co-authorship network and bibliographic coupling with institutions, authors and countries based on the research output of Nephrology. Using the Web of Science core collection citation database from the Clarivate Analytics, a total number of 2626 literature found by the researcher and the total citations in global level was 11, 993 and the total cited references were 84, 129 and the total authors was 10, 846, total number of core journals was 595 and institutions (3494), Institution with subdivision (6630), and 108 total countries were participated in the study. The scientometric indicators and computer software applied for the present study to retrieve appropriate results on Nephrology research such as Average citation per paper (ACPP), Exponential growth rate (EGR), VOS viewer mapping software, HistCite software, Microsoft Excel and Microsoft Word to bring out fruitful results.
\end{abstract}

Keywords: Nephrology, Bibliographic Coupling, Co-authorship Network, VOS viewer, Scientometrics, Bibliometrics

\section{BACKGROUND}

Scientometrics is the popular metric study connected with publication analysis using various quantitative and qualitative techniques and other statistical tools and methods. Bibliographic coupling is one of the topics in scientometric study in Library and Information Science. 
Bibliographic coupling is used to identify a similarity measure like co-citation to establish a similarity relationship between research publications. Bibliographic coupling occurs when two works reference a common third work in their bibliographies. Bibliographic coupling is used in a wide variety of subjects and fields, and it helps researchers find related research done in the past (1). In 1973, Kessler devised the term Bibliographic coupling is "to know the number of common references cited in two articles and represents the degree of similarity of contents of the cited item or paper". Bibliographic coupling network can be built for various units of analysis like authors, journals and scientific publications whereas Co-authorship networks can be made for various units of analysis such as authors, productive countries, and organizations using full and fractional counting. In 1973, Small $(1973,1985)$ introduced the concept of co-citation analysis to map the clusters of related documents. He defined as, "the number times the two papers are cited together in subsequent literature determines the co-citation strength of the two cited papers".

Nephrology is the up-and-coming thrust area in medicine which is associated with kidney. The phrase "Nephrology" derived from Greek word 'nephros' means 'kidney', combined with the suffix -logy, means "the study of" is a specialty of medicine and pediatrics that concerns itself with the kidneys and it is associated with the study of normal kidney function and kidney disease, the preservation of kidney health, and the treatment of kidney disease, from diet and medication to renal replacement therapy (Wikipedia). According to Science.org website, the word "nephrology" pronounced for the first time in a conference which was held on from $1^{\text {st }}$ to $4^{\text {th }}$ September 1960 at the "Premier Congress International de Néphrologie" in Evian and Geneva during the first meeting of the International Society of Nephrology (https://www.science. org.au/). In this present study, researcher tried to identify the Bibliographic coupling and coauthorship networks of scientific publications in the field of Nephrology during the period between 2014 and 2018 .

\section{RELATED WORK}

A number of studies have been conducted in the field of Medicine but there are few studies carried out in the topic on Nephrology. Velmurugan (2018) analyzed the research out of Nephrology to examine the various aspects and found regarding document type and language wise distribution, ranking of Journals, authorship pattern, degree of collaboration and most productive keywords during 2011-2016. He found United States had ranked the first publishing scientific papers and followed by Italy ranked the second. He also noted the highest papers were found in the year 2014 and the research articles had ranked first position among other medium of communication. O'Connor; Nason and O'Brien (2017) examined to find out the Ireland's contribution to urology and nephrology research in the new millennium by way of bibliometric analysis. They identified that the Ireland's contribution of research publications were very low $(0.51 \%)$ compare with global output in the research of urology and nephrology. Sweileh et al (2014) investigated a study to analyze the research performance in the field of urology and nephrology in Arab countries through bibliometric techniques. They found 3076 research output in urology and nephrology subject from 104 core journals. Based on the results, they suggested that more efforts were needed through some other Arab countries to bridge the gap in the field of urology and nephrology. Moreover, in the field of Nephrology (1996, 2000, 2006, 2007, 2012), Urology and Nephrology (2003, 2012) and Chronic kidney (1972, 2004, 2012) had conducted already in different countries by different researchers and eminent scholars throughout the globe. 


\section{METHODS}

The data retrieved in the present study were based on the ISI Web of Science, which is one of the global level largest citation databases of peer reviewed publications. The search keywords "Nephrology" phrase used and selected in the field of topic and the time span was from 2013 to 2018 as six years period of study. Using the Web of Science core collection from the Clarivate Analytics, a total number of 2626 literature found by the researcher and the total citations in global level was 11,993 and the total cited references were 84, 129 and the total authors was 10 , 846, total number of core journals was 595 and institutions (3494), Institution with subdivision (6630), and 108 total countries were participated in the study. Further, the database counted a total number of publications, total number of citations, and their value of ACPP (Average citation per paper) and Exponential growth rate (ECR) to measure the quantifying research performance. Data of nephrology was exported to HistCite software and then transferred to Microsoft Word for further analysis. Moreover, VOS viewer software used for data visualization in terms of co-authorship with authors, institutions, and countries, and Bibliographic coupling with authors, institutions, and countries.

\section{OBJECTIVES}

The main objective was to identify the co-authorship network and bibliographic coupling with institutions, authors and countries based on the research output of Nephrology. The other objectives were:

* To find out the various kinds of medium of publications

* To explore the year-wise contribution of literature

* To know about the language wise contribution

* To investigate the collaborative research in the field of Nephrology

* To observe the total link strength of each item of Nephrology

\section{INDICATORS AND SOFTWARE}

The following scientometric indicators and computer software applied for the present study to retrieve appropriate results on Nephrology research.

\footnotetext{
* Average citation per paper (ACPP)

* Exponential growth rate (EGR)

* VOS viewer mapping software

* HistCite software

* Microsoft Excel and

* Microsoft Word
} 


\section{ANALYSIS}

\section{Medium of Communication}

Communication is the unique characteristics to share information to right person to right way in right time. As far as the publications concerned, the medium of communication has varied depends upon the nature of publications. Here, there are 11 types of manuscripts found through the nephrology literature. Table 1 discusses the medium type, total records, and their share and total citations in global level and even average citation per paper also evaluated. Out of 2626 publications, a large amount of 1826 (69.5\%) articles were research papers and its total global citations were 9151 and its average citation per paper was 5.01. The next productive medium of communication was review papers with $304(11.6 \%)$, and its average citation per paper was 7.35 followed by Editorial Materials (8.8\%) and Meeting Abstracts (6.3\%). The other items were below hundred of records and the results show that original research articles were predominant and the researchers and scientists preferred to publish research papers rather than other items like reviews etc.

Table.1. Medium of Communication

\begin{tabular}{|c|l|c|c|c|c|}
\hline S. No & \multicolumn{1}{|c|}{ Medium Type } & Total Records & Total Percent & TGCS & ACPP \\
\hline 1 & Articles & 1826 & 69.5 & 9151 & 5.01 \\
\hline 2 & Reviews & 304 & 11.6 & 2234 & 7.35 \\
\hline 3 & Editorial Materials & 231 & 8.8 & 461 & 1.99 \\
\hline 4 & Meeting Abstracts & 165 & 6.3 & 1 & 0.006 \\
\hline 5 & Letters & 41 & 1.6 & 31 & 0.75 \\
\hline 6 & Article; Proceedings Papers & 25 & 1.0 & 70 & 2.80 \\
\hline 7 & News Items & 11 & 0.4 & 14 & 1.27 \\
\hline 8 & Biographical-Items & 9 & 0.3 & 2 & 0.22 \\
\hline 9 & Corrections & 9 & 0.3 & 1 & 0.11 \\
\hline 10 & Article; Book Chapters & 3 & 0.1 & 18 & 6.00 \\
\hline 11 & Review; Book Chapters & 2 & 0.1 & 10 & 5.00 \\
\hline \multicolumn{2}{r|}{ Total } & 2626 & 100 & 11993 & 4.57 \\
\hline
\end{tabular}

\section{Growth of publications}

Researchers scrutinized the chorology wise publication share during 2013-2018. They have measured different ways like total records, total local citation score, total global citation score, Exponential growth rate and average citation per paper etc. Table 2 (Ch.1) indicates that out of 2626 literature output, the vast amount of research articles published in 2016 and the least amount of papers published in 2013. They have also evaluated an average citation per paper and found the range was from 0.27 to 8.11 and the highest average citation per paper was 8.11 in 2013 with 382 (14.6\%), and its global citation score was 3098. As far as the exponential growth rate concerned, 1.18 was the highest rate by both years 2015 and 2018 respectively. The analysis shows that there is a fluctuation trend in the growth of publications on Nephrology during 20132018. 
Table.2. Growth of publications

\begin{tabular}{|c|c|c|c|c|c|c|c|}
\hline S. No & Year & Records & Percent & TLCS* & TGCS* & EGR* & ACPP \\
\hline 1 & 2013 & 382 & 14.6 & 365 & 3098 & - & 8.11 \\
\hline 2 & 2014 & 400 & 15.3 & 411 & 2879 & 1.05 & 7.19 \\
\hline 3 & 2015 & 473 & 18.0 & 306 & 3304 & 1.18 & 6.98 \\
\hline 4 & 2016 & 510 & 19.5 & 185 & 1797 & 1.07 & 3.52 \\
\hline 5 & 2017 & 395 & 14.8 & 88 & 789 & 0.77 & 1.99 \\
\hline 6 & 2018 & 466 & 17.8 & 23 & 126 & 1.17 & 0.27 \\
\hline & 10 & 2626 & 100 & 1378 & 11993 & 5.25 & 4.57 \\
\hline
\end{tabular}

*TLCS - Total Local Citation Score, *TGCS - Total Global Citation Score, *EGR - Exponential Growth Rate

Chart 01. Exponential Growth Rate

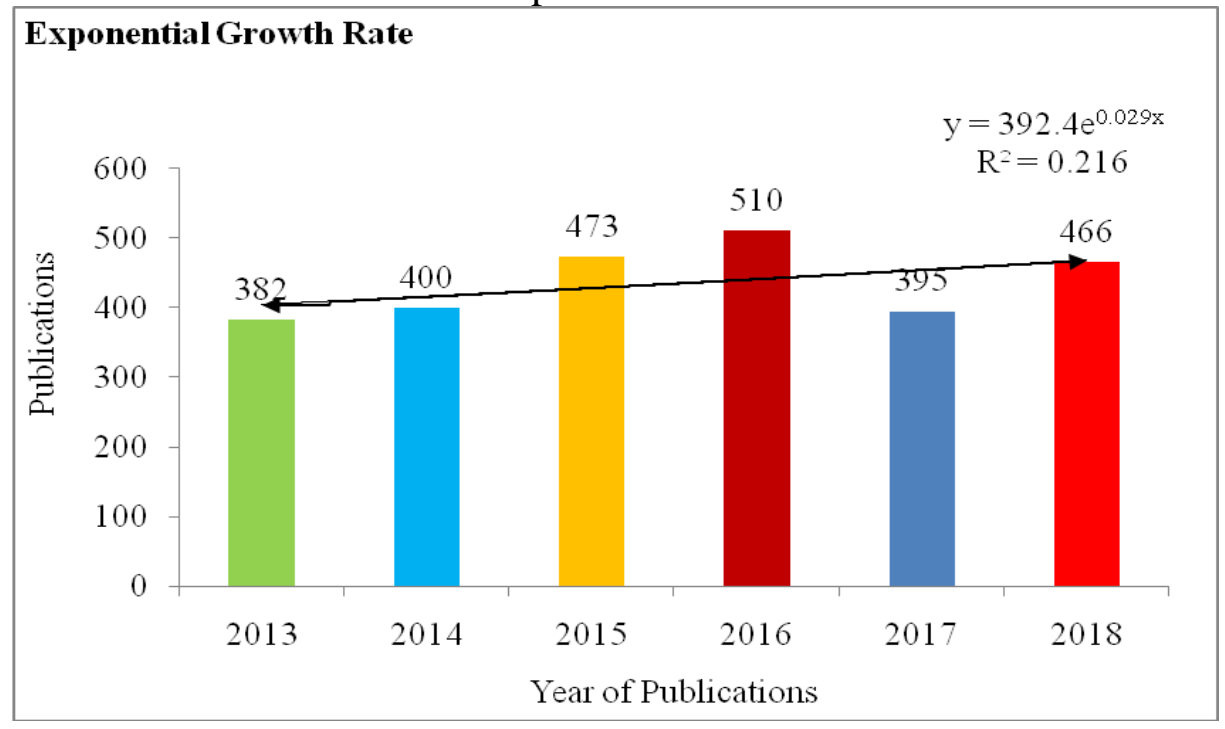

Exponential growth is a specific way that the quantity may increase over time. It occurs when the instantaneous rate of change of a quantity with respect to time is proportional to the quantity itself (Wikipedia, 2020). Here, Exponential Growth Rate has also measured and found in nephrology research publications between 2013 and 2018. Researchers identified the highest growth rate was 1.18 during 2015 with 473 scientific papers and also noted that the Exponential Growth Rate was found to be 5.25. It also calculated the exponential growth rate using Microsoft Excel (Chart.1) and found the $\mathrm{y}$ value is $392.4 \mathrm{e}^{0.029 \mathrm{x}}$ and $\mathrm{R}^{2}$ value is 0.216 . It shows that the increasing trend in the field of Nephrology over the period of study.

\section{Distribution by Verbal Communication}

Verbal communication is a part and parcel in our day today life. This is an essential indicator in the field of Library and Information Science, particularly in Scientometrics in order to determine the highest growth of papers published in which language and country. Here, researchers examined the language wise publication productivity and its share of publications and an average citation per paper in Nephrology research. Table 00 illustrates that out of 12 languages, the greatest number of 2387 literature output (91.0\%) published in English language as expected. 
The next productive papers (120) were published in Spanish language, and followed by French with 63 (2.3\%) publications. The papers below 5 published by Russian, Italian, Serbian, Korean, Turkish and Rumanian languages and shows that the language people were not aware about the literature trend and publication growth.

Table 3. Distribution by Verbal Communication

\begin{tabular}{|c|c|c|c|c|}
\hline Language & $\begin{array}{c}\text { Total } \\
\text { Records }\end{array}$ & $\begin{array}{l}\text { Total } \\
\text { Percent }\end{array}$ & $\begin{array}{c}\text { Total } \\
\text { Citations }\end{array}$ & $\begin{array}{l}\text { Average Citation } \\
\text { Per Paper }\end{array}$ \\
\hline English & 2387 & 91.0 & 11713 & 4.91 \\
\hline Spanish & 120 & 4.6 & 228 & 1.90 \\
\hline French & 63 & 2.3 & 23 & 0.36 \\
\hline German & 30 & 1.1 & 17 & 0.56 \\
\hline Portuguese & 6 & 0.2 & 3 & 0.5 \\
\hline Polish & 5 & 0.2 & 3 & 0.6 \\
\hline Russian & 4 & 0.2 & 0 & 0 \\
\hline Italian & 3 & 0.1 & 0 & 0 \\
\hline Serbian & 3 & 0.1 & 1 & 0.33 \\
\hline Korean & 2 & 0.1 & 3 & 1.50 \\
\hline Turkish & 2 & 0.1 & 2 & 1.00 \\
\hline Rumanian & 1 & 0.0 & 0 & 0 \\
\hline Total & 2626 & 100 & 11996 & 0.97 \\
\hline
\end{tabular}

\section{Co-authorship network with authors}

Table 4 (M.1) points out the authors' network visualization mapping of co-authorship network in Nephrology research. A total of 10, 872 prolific authors produced 2626 papers, and the maximum number of authors per paper was 25 and the minimum number of 5 with full counting method used. Out of 10872, only 181 authors met the thresholds. Here, there were 1555 total link strength and thirteen clusters with 161 items, in which cluster 1 (26 items), cluster 2 (23 items), cluster 3 (20 items), cluster 4 (17 items), cluster 5 (14 items), cluster 6 (13 items), cluster 7 (11 items), cluster 8 (10 items), cluster 9 ( 8 items), cluster 10 (6 items), cluster 11,12 and 13 (each 5 items). It is witnessed that a number of 27 articles of "Jager, Kitty, J" and WITH 98 total link strength and "Sinha, Manish D" have 83 total link strength with 16 articles found through analysis. It represents that the authors involved in this research had a strong collaboration each other in producing research publications. 
Table. 4. Co-authorship network with authors

\begin{tabular}{|l|r|r|r|}
\hline \multicolumn{1}{|c|}{ Author } & Documents & Citations & $\begin{array}{l}\text { Total link } \\
\text { strength }\end{array}$ \\
\hline jager, kitty j. & 27 & 163 & 98 \\
\hline sinha, manish d. & 16 & 36 & 83 \\
\hline inward, carol & 13 & 35 & 80 \\
\hline lewis, malcolm & 11 & 25 & 74 \\
\hline casula, anna & 9 & 17 & 67 \\
\hline zoccali, carmine & 24 & 184 & 66 \\
\hline maxwell, heather & 8 & 17 & 65 \\
\hline o'brien, catherine & 8 & 17 & 65 \\
\hline pruthi, rishi & 8 & 17 & 65 \\
\hline schaefer, franz & 15 & 77 & 64 \\
\hline de nicola, luca & 16 & 109 & 61 \\
\hline minutolo, roberto & 16 & 109 & 61 \\
\hline tse, yincent & 8 & 19 & 59 \\
\hline conte, giuseppe & 15 & 108 & 58 \\
\hline van stralen, karlijn j. & 11 & 57 & 58 \\
\hline verrina, enrico & 10 & 39 & 53 \\
\hline braddon, fiona & 7 & 13 & 51 \\
\hline \hline
\end{tabular}

Mapping. 1. Co-authorship network with authors

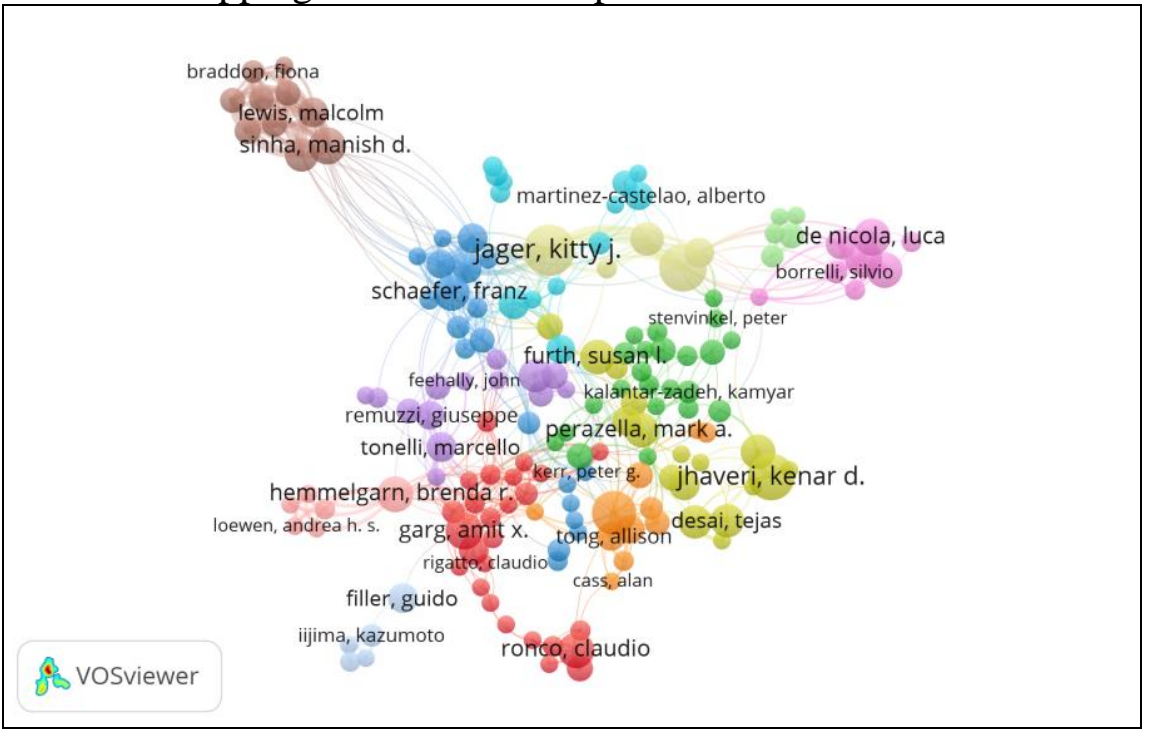

\section{Co-authorship network with countries}

Based on the data, full counting co-authorship network has been counted using VOSviewer software. In mapping, each circle denotes a country and the dimension of the circle characterize the number of scientific publications of corresponding countries in the field of Nephrology. The range between circles shows the potency of co-authorship link between the corresponding countries whereas the nearer two circles are situated to each other, the stronger the co-authorship link connecting the countries.

Table 5 (M.2) represents that the data visualization of the countries which were involved in this Nephrology research using full counting records. A total of 112 countries produced 2626 publications, and the maximum number of countries per document was 25 selected and the 
minimum number of 5 countries was selected, and 61 countries met the threshold level. In this mapping, there were seven clusters with 61 items, cluster 1 (22 items), cluster 2 (20 items), cluster 3 ( 7 items), cluster 4 and 5 (each 4 items), cluster 6 and 7 (each 2 items. It is evident that United States had 428 total link strength with 869 articles and followed by Italy had 395 total link strength with 217 papers. It is also measured the countries with the greatest total link strength has been calculated as 2729. The results showed that United States had raked first as more number of papers co-authored with other scientists and scholars and it indicates their relationship in Nephrology research.

Table.5. Co-authorship network with countries

\begin{tabular}{|l|r|r|r|}
\hline \multicolumn{1}{|c|}{ Country } & Documents & Citations & $\begin{array}{l}\text { Total link } \\
\text { strength }\end{array}$ \\
\hline usa & 869 & 5330 & 428 \\
\hline italy & 217 & 1657 & 395 \\
\hline england & 178 & 1572 & 393 \\
\hline germany & 169 & 1418 & 357 \\
\hline france & 150 & 955 & 319 \\
\hline netherlands & 90 & 1087 & 280 \\
\hline belgium & 59 & 466 & 261 \\
\hline canada & 206 & 1912 & 240 \\
\hline spain & 144 & 838 & 229 \\
\hline australia & 148 & 802 & 210 \\
\hline poland & 53 & 256 & 155 \\
\hline turkey & 86 & 243 & 136 \\
\hline austria & 30 & 240 & 133 \\
\hline sweden & 46 & 446 & 125 \\
\hline brazil & 66 & 336 & 124 \\
\hline greece & 28 & 250 & 114 \\
\hline czech republic & 17 & 219 & 111 \\
\hline denmark & 29 & 222 & 104 \\
\hline
\end{tabular}

Mapping. 2. Co-authorship network with countries

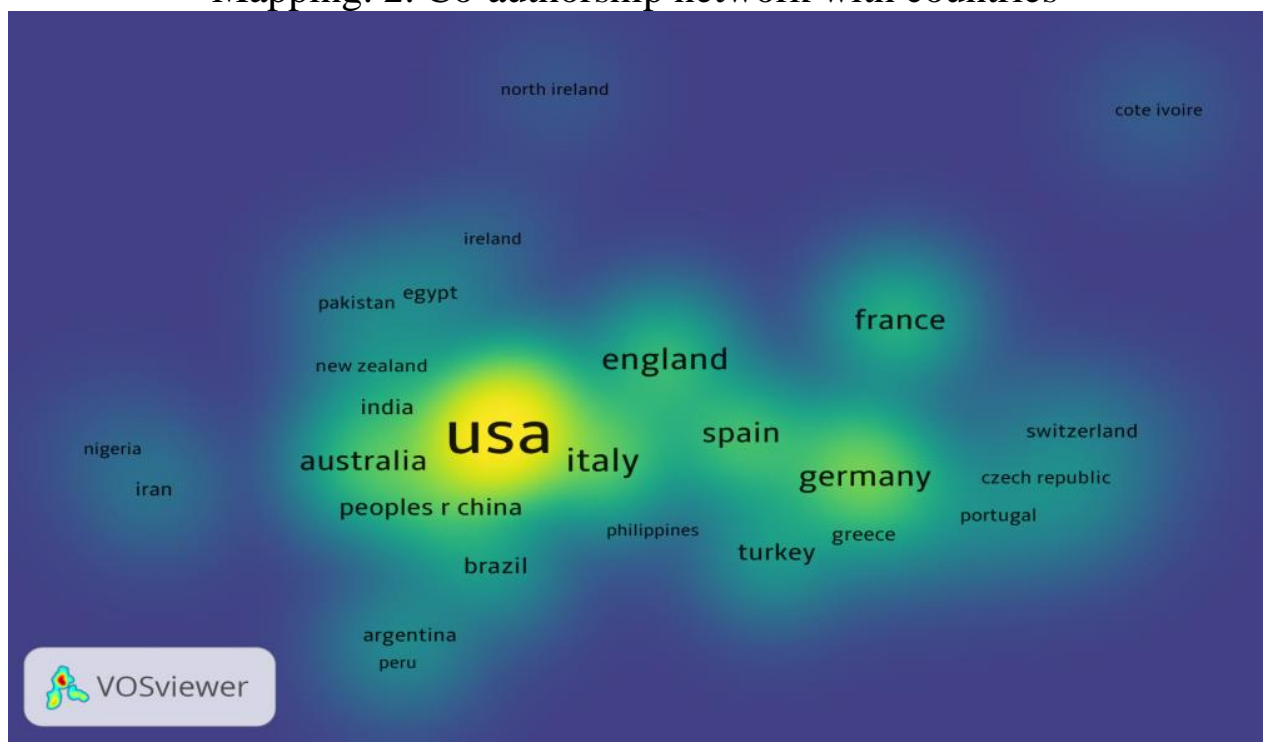




\section{Bibliographic Coupling with Sources}

It explains the source based network visualization mapping of bibliographic coupling on Nephrology publications. A total of 595 prolific sources produced research articles, and the minimum number of 5 with full counting method was selected, and only 63 sources met the thresholds. For each of the 63 sources, the total strength of the bibliographic coupling links with other sources has been calculated. The sources of this research, the greatest total link strength (TLS) was calculated and found 46716 TLS and 7 clusters with 63 items.

Here, there were seven clusters and the first cluster 1 (16 items), cluster 2 (14 items), cluster 3 (14 items), cluster 4 (7 items), cluster 5 (6 items), cluster 6 (5 items), and cluster 7 with one item. It shows that the maximum number of total link strength was 10017 with 140 articles with 1324 citations by the source journal of "Clinical Journal of the American Society of Nephrology" and ranked first and followed by "American Journal of Kidney Diseases" ranked second with 9787 TLS with 145 research articles. It depicts that the two source journals had a strong collaboration each other in publishing scholarly papers (Table 6, M.3).

Table.6. Bibliographic Coupling with Sources

\begin{tabular}{|l|r|r|r|}
\hline \multicolumn{1}{|c|}{ Source } & Documents & Citations & $\begin{array}{c}\text { Total link } \\
\text { strength }\end{array}$ \\
\hline clinical journal of the american soc... & 140 & 1324 & 10017 \\
\hline american journal of kidney diseases & 145 & 1101 & 9787 \\
\hline nephrology dialysis transplantation & 142 & 765 & 7748 \\
\hline kidney international & 93 & 899 & 5721 \\
\hline nefrologia & 79 & 230 & 4886 \\
\hline bmc nephrology & 46 & 141 & 4704 \\
\hline advances in chronic kidney disease & 42 & 122 & 3381 \\
\hline journal of nephrology & 75 & 140 & 2952 \\
\hline pediatric nephrology & 130 & 404 & 2846 \\
\hline nephrology & 66 & 101 & 2609 \\
\hline nephron clinical practice & 51 & 221 & 2583 \\
\hline seminars in dialysis & 57 & 265 & 2530 \\
\hline plos one & 25 & 106 & 2297 \\
\hline clinical and experimental nephrolo... & 29 & 195 & 2163 \\
\hline journal of the american society of ... & 24 & 269 & 2125 \\
\hline nature reviews nephrology & 23 & 195 & 1977 \\
\hline renal failure & 49 & 87 & 1809 \\
\hline american journal of nephrology & 16 & 76 & 1788 \\
\hline clinical nephrology & 32 & & 1634 \\
\hline
\end{tabular}


Mapping. 3. Bibliographic Coupling with Sources

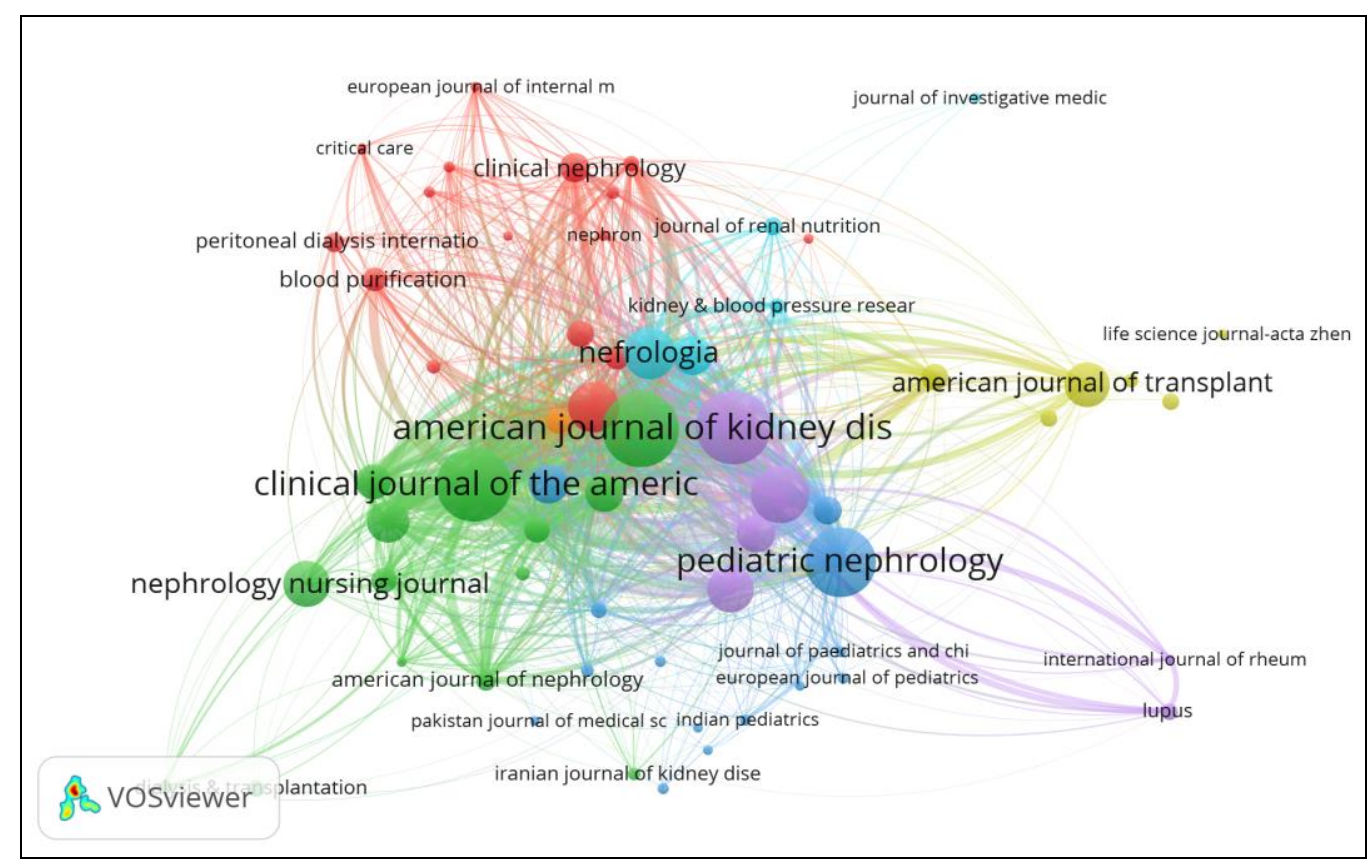

\section{Bibliographic Coupling with authors}

Table 7 (M.4) display the authors' density visualization mapping of bibliographic coupling on Nephrology research. A total of 10872 prolific authors produced research papers, and the minimum number of 5 with full counting method was selected, and only 181 authors met the thresholds. Here, there were 1, 34, 153 total link strength and ten clusters with 181 items, in which cluster 1 (42 items), cluster 2 (32 items), cluster 3 ( 27 items), cluster 4 (23 items), cluster 5 (15 items), cluster 6 (13 items), cluster 7 (10 items), cluster 8 ( 9 items), cluster 9 (6 items) and cluster 10 (4 items). For each of the 181 authors, the total strength of the bibliographic coupling links with other authors has been calculated. It is witnessed that a number of 16 articles of "de nicola, luca" and "minutola, roberto" both coupled 5769 times and shows as total link strength and followed by 15 research publications of "conte, giuseppe" coupled 5608 times. Therefore, the above authors had a strong collaboration among them in producing research publications. 
Table. 7. Bibliographic Coupling with authors

\begin{tabular}{|l|r|r|r|}
\hline \hline \multicolumn{1}{|c|}{ Author } & Documents & Citations & $\begin{array}{c}\text { Total link } \\
\text { strength }\end{array}$ \\
\hline de nicola, luca & 16 & 109 & 5769 \\
\hline minutolo, roberto & 16 & 109 & 5769 \\
\hline conte, giuseppe & 15 & 108 & 5608 \\
\hline hemmelgarn, brenda r. & 15 & 212 & 5127 \\
\hline jager, kitty j. & 27 & 163 & 4663 \\
\hline chiodini, paolo & 11 & 88 & 4550 \\
\hline patel, uptal d. & 9 & 116 & 4362 \\
\hline schaefer, franz & 15 & 77 & 3986 \\
\hline kalantar-zadeh, kamyar & 7 & 56 & 3846 \\
\hline whaley-connell, adam t. & 5 & 38 & 3787 \\
\hline zoccali, carmine & 24 & 184 & 3683 \\
\hline tonelli, marcello & 11 & 143 & 3619 \\
\hline borrelli, silvio & 7 & 83 & 3417 \\
\hline craig, jonathan c. & 21 & 69 & 3370 \\
\hline garg, amit x. & 16 & 242 & 3264 \\
\hline navaneethan, sankar d. & 5 & 115 & 3229 \\
\hline martinez-castelao, alberto & 10 & 30 & 3123 \\
\hline
\end{tabular}

Mapping. 4. Authors density visualization of bibliographic coupling

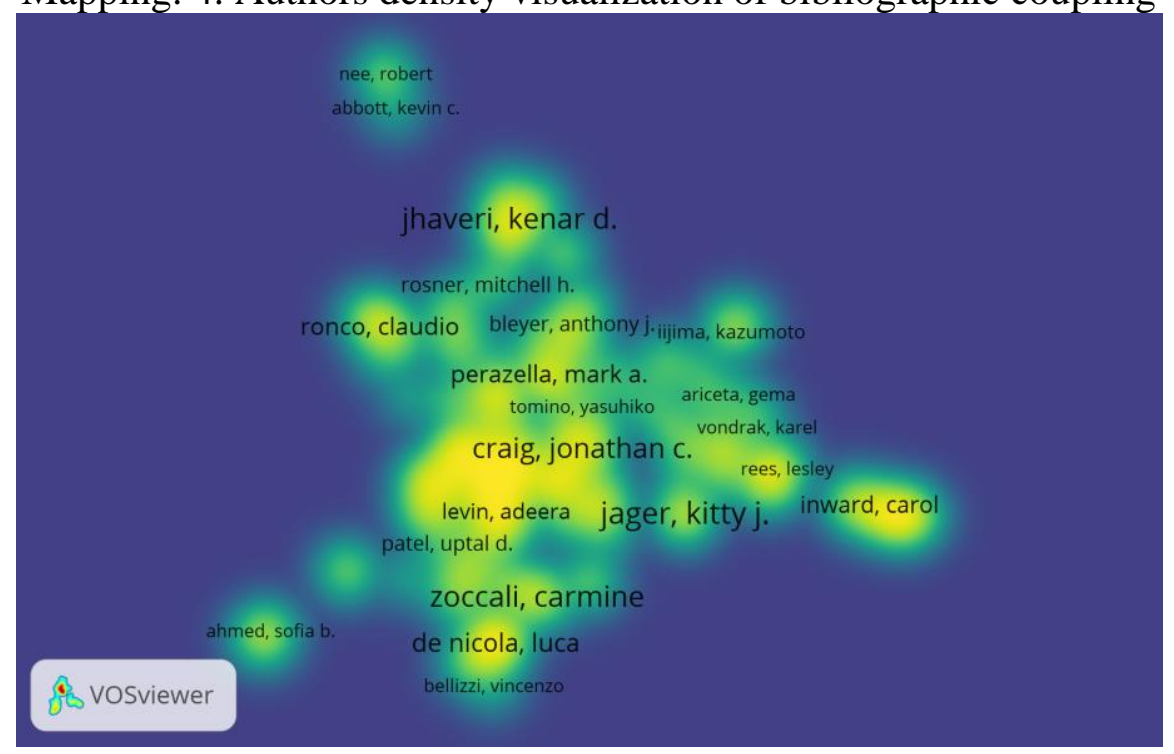

\section{Bibliographic Coupling with Organizations}

Table 8 (M.5) shows the mapping of bibliographic coupling with institutions on Nephrology research. A total of 323 institutions involved producing research output, and the minimum number of 5 organizations was selected, and 323 institutions met the threshold level. In this mapping, there were 5, 45, 681 total link strength and eight clusters with 323 documents, in which cluster 1 with 79 items and cluster 2 with 65 items, cluster 3 with 54 items, cluster 4 with 42 items, and cluster 5 with 35 items and cluster 6 with 26 items, cluster 7 with 16 and cluster 8 with 6 items. For each of the 323 institutions, the total strength of the bibliographic coupling links with other institutions has been calculated. It is evident that the majority of 61 articles of "University of Toronto" coupled 27, 401 times and shows as total link strength and followed by 43 research publications of "University of Alberta" coupled 23, 765 times. Therefore, both institutions had a good relationship in terms of scholarly publications. 
Table. 8. Bibliographic Coupling with Organizations

\begin{tabular}{|l|r|r|r||}
\hline \multicolumn{1}{|c|}{ Organization } & Documents & Citations & $\begin{array}{c}\text { Total link } \\
\text { strength }\end{array}$ \\
\hline univ toronto & 61 & 453 & 27401 \\
\hline univ alberta & 43 & 493 & 23765 \\
\hline univ calgary & 39 & 394 & 22392 \\
\hline univ washington & 29 & 286 & 17294 \\
\hline univ western ontario & 38 & 497 & 16800 \\
\hline duke univ & 28 & 229 & 15886 \\
\hline univ ottawa & 25 & 208 & 15666 \\
\hline univ manitoba & 22 & 108 & 13682 \\
\hline univ british columbia & 28 & 181 & 13228 \\
\hline mayo clin & 43 & 615 & 13156 \\
\hline yale univ & 28 & 187 & 12027 \\
\hline univ sydney & 47 & 137 & 11681 \\
\hline emory univ & 35 & 134 & 11490 \\
\hline harvard univ & 46 & 393 & 11320 \\
\hline univ calif san francisco & 29 & 191 & 11163 \\
\hline univ missouri & 11 & 104 & 11137 \\
\hline mcmaster univ & 18 & 139 & 10821 \\
\hline northwestern univ & 23 & 294 & 10562 \\
\hline univ pittsburgh & 21 & 10522 \\
\hline
\end{tabular}

Mapping. 5. Bibliographic Coupling with Organizations

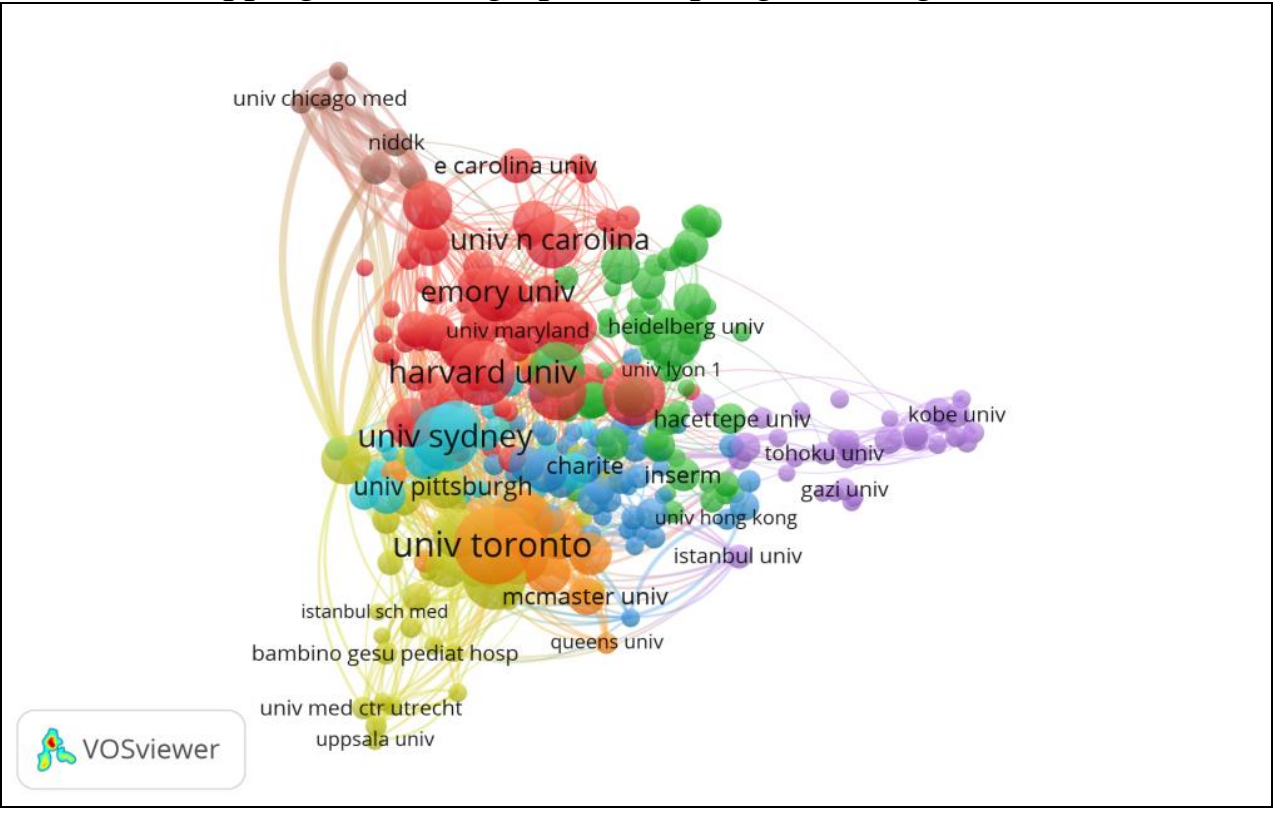

\section{Bibliographic Coupling with countries}

Table 9 (M.6) indicates the mapping of bibliographic coupling with countries on Nephrology research. A total of 112 countries participated to publish publications, and the minimum number of 5 countries was selected, and 61 countries met the threshold level. In this mapping, there were five clusters with 61 items, cluster 1and 2 with 24 items, cluster 3 with 10 items, cluster 4 with 2 items, and cluster 5 with only one item. For each of the 61 countries, the total strength of the bibliographic coupling links with other countries has been measured. It is seen from the below table that more than 10,000 (Scotland) total link strength to 95, 436 (United States) total link strength. It shows a huge amount of 95, 436 times coupled with 869 documents with 5, 330 
citations in United States and followed by 49, 215 times coupled with 217 documents with 1, 657 citations by Italy. It is also measured the countries with the greatest total link strength has been calculated as 3, 37, 602. It determines that both countries cited more papers to strengthen their relationship in Nephrology research.

Table. 9. Bibliographic Coupling with countries

\begin{tabular}{|l|r|r|r|}
\hline \multicolumn{1}{|c|}{ Country } & Documents & Citations & $\begin{array}{c}\text { Total link } \\
\text { strength }\end{array}$ \\
\hline usa & 869 & 5330 & 95436 \\
\hline italy & 217 & 1657 & 49215 \\
\hline canada & 206 & 1912 & 45954 \\
\hline england & 178 & 1572 & 41376 \\
\hline france & 150 & 955 & 34802 \\
\hline germany & 169 & 1418 & 32198 \\
\hline spain & 144 & 838 & 30760 \\
\hline belgium & 59 & 466 & 28865 \\
\hline netherlands & 90 & 1087 & 27105 \\
\hline australia & 148 & 802 & 26440 \\
\hline japan & 107 & 1303 & 18015 \\
\hline brazil & 66 & 336 & 17250 \\
\hline sweden & 46 & 446 & 16130 \\
\hline austria & 30 & 240 & 15166 \\
\hline turkey & 86 & 243 & 13324 \\
\hline greece & 28 & 250 & 12968 \\
\hline peoples r china & 87 & 711 & 12198 \\
\hline poland & 53 & 256 & 11258 \\
\hline scotland & 34 & 279 & 10391 \\
\hline
\end{tabular}

Mapping 6. Bibliographic Coupling with countries

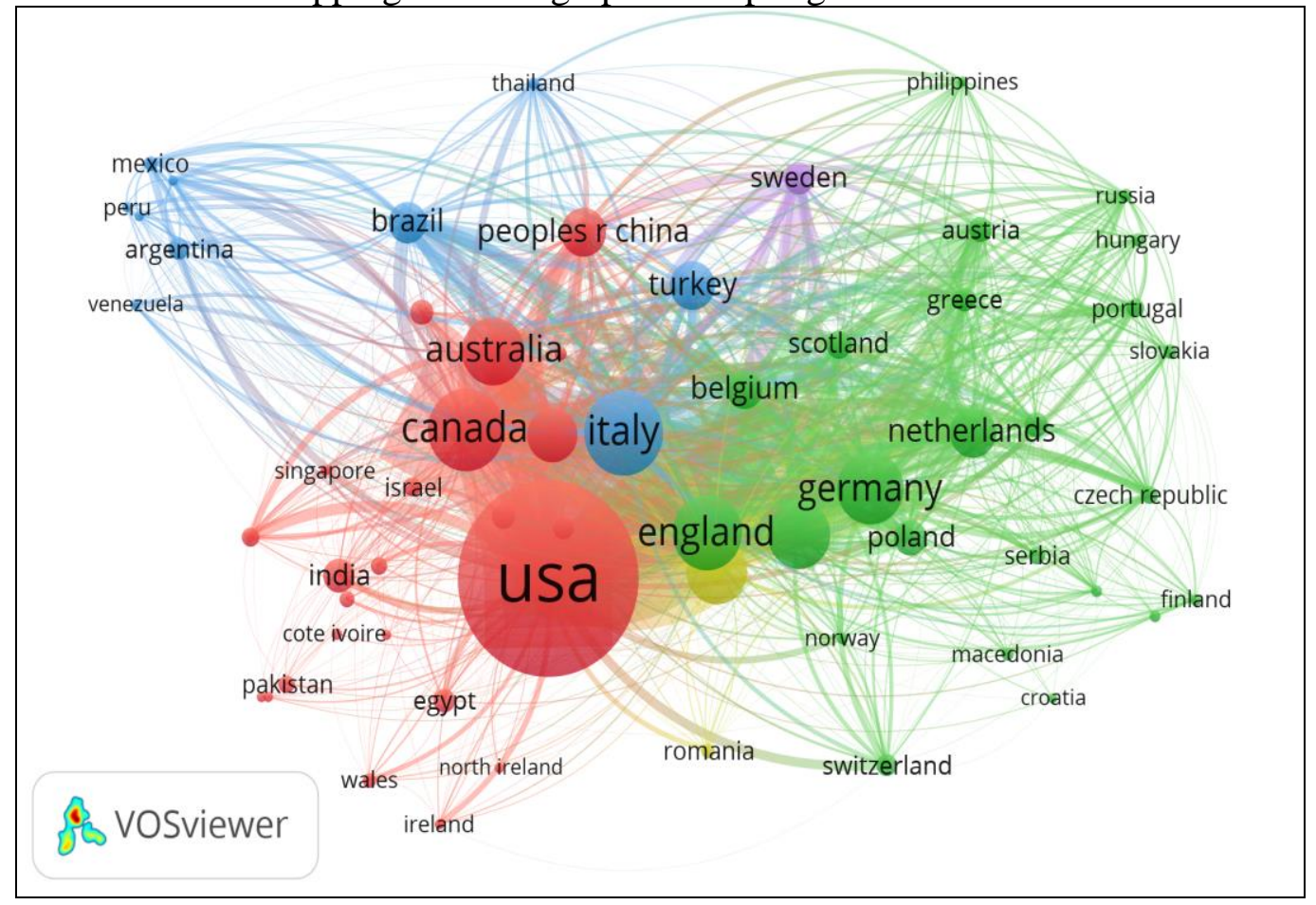




\section{Findings}

* It is examined that an average citation per paper in Nephrology research and found the highest average citation per paper was 8.11 in 2013.

* The majority of the exponential growth rate (1.18) which was the highest rate by both years 2015 and 2018 respectively.

* It is found that the greatest number of 2387 literature output (91.0\%) published in English language.

* The maximum number of total link strength was 10017 with 140 articles with 1324 citations by the source journal of "Clinical Journal of the American Society of Nephrology" and ranked first and followed by "American Journal of Kidney Diseases" ranked second with 9787 TLS with 145 research articles.

* The major portion of 16 articles of "de nicola, luca" and "minutola, roberto" both coupled 5769 times and shows as total link strength and followed by 15 research publications of "conte, giuseppe" coupled 5608 times.

* It is found that the majority of 61 articles of "University of Toronto" coupled 27, 401 times and shows as total link strength and followed by 43 research publications of "University of Alberta" coupled 23, 765 times.

* It noted a huge amount of 95, 436 times coupled with 869 documents with 5, 330 citations in United States and followed by 49, 215 times coupled with 217 documents with 1, 657 citations by Italy.

\section{Conclusion}

Citation analysis is one of the most effective indictor in scholarly research. According to Gooden (2001), citation analysis is "an excellent way to determine the information resources that researchers use in a field, the volume of research in the area, and the patterns of citation and authorship". Citation analysis is used as guide for researchers, information scientists, librarians and other professionals on how to support research in the course of acquisition of the core journals and to know about the most prolific authors in LIS field. Meho (2007) pointed out that citation analysis is "a branch of information science in which researchers study the way articles in a scholarly field are accessed and referenced by others." The present study focuses on the bibliographic coupling and collaborative research by way of VOSViewer visualization mapping software. This software has been applied to identify the co-authorship network and bibliographic coupling with institutions, authors and countries based on the research output of Nephrology. So, we can suggest that this study will help to nephrology professionals to know the collaborative work and ideas of others. People also count citations of a paper as an indication of how significant or influential the paper has. Moreover, this study will tremendously useful to know about the amount of research made by researchers which strengthens their work by through outside support. 


\section{Reference}

1. Allison MM. (2006). Mapping the literature of nephrology nursing. J Med Libr Assoc, 94, 74-79.

2. Dunn MD, Portis AJ, Shalhav AL, Elbahnasy AM, Heidorn C, McDougall EM, Clayman RV. (2000). Laparoscopic versus open radical nephrectomy: a 9-year experience. J Urol, 164, 1153-1159.

3. Einollahi, B. (2007). Nephrology research output in Iran in a decade. Iran J Kidney Dis, $1,57-62$.

4. Eknoyan G, Lameire N, Barsoum R, Eckardt KU, Levin A, Levin N, Locatelli F, MacLeod A, Vanholder R, Walker R, Wang H. (2004). The burden of kidney disease: improving global outcomes. Kidney Int, 66, 1310-1314.

5. Farag YM, Kari JA, Singh AK. (2012). Chronic kidney disease in the Arab world: a call for action. Nephron Clin Pract, 121, c120-c123.

6. Gooden, A. M. (2001), "Citation Analysis of Chemistry Doctoral Dissertations: An Ohio State University Case Study," Issues in Science and Technology Librarianship, 32, 1-16, available at: http://findarticles.com/p/articles/mi.

7. Kessler, M.M. (1963). Bibliographic coupling between scientific papers. American Documentation, 14(1), 10-25.

8. Kou J, Zhang Y, Zhang XG, Liang S, Cai GY, Chen XM. (2012). Clinical research promotes development of nephrology in China: an analysis of 20 years of scientific publications. Ren Fail, 34, 472-479.

9. Meho, L. I. (2007). The Rise and Rise of Citation Analysis. Physics World, 20 (1), 3236.

10. Menon M, Hemal AK, Tewari A, Shrivastava A, Shoma AM, El-Tabey NA, Shaaban A, Abol-Enein H, Ghoneim MA. (2003). Nerve-sparing robot-assisted radical cystoprostatectomy and urinary diversion. BJU Int, 92, 232-236.

11. Nephrology, accessed at: https://www.science. org.au/

12. Nephrology, Wikipedia, accessed at: https://en.wikipedia.org/wiki/Nephrology

13. O’Connor EM, Nason GJ, O'Brien MF (2017). Ireland's contribution to urology and nephrology research in the new millennium: a bibliometric analysis. Irish Journal of Medical Science, 186 (2), 371-377. Doi: 10.1007/s11845-016-1485-8. 
14. Saied H, Dupré A, Lacki W, Paley D. (1972). Pelvic and lower lumber ectopy of kidney in children - with 7 case reports. Ann Urol, 6, 227-233.

15. Schena FP, Zoccali C. (1996). Bibliometric analysis of the scientific productivity of the Italian Society of Nephrology for a period of five consecutive years (1990-1994). Nephrol Dial Transplant, 11, 2359-2360.

16. Small, H. (1973). Co-citation in the scientific literature: A new measure of the relationship between two documents. Journal of the American Society for Information Science, 24(4), 265-269.

17. Small, H., \& Sweeney, E. (1985). Clustering the Science Citation Index using cocitations: I. A comparison of methods. Scientometrics, 7(3-6), 391-409.

18. Sweileh WM, Zyoud SH, Al-Jabi SW, Sawalha AF (2014). Assessing urology and nephrology research activity in Arab countries using ISI web of science bibliometric database. BMC research notes; 7:258. Doi: 10.1186/1756-0500-7-258.

19. Van Eck, N.J., \& Waltman, L. (2010). Software survey: VOSviewer, a computer program for bibliometric mapping. Scientometrics, 84(2), 523-538.

20. Velmurugan, C. (2020). Mapping of Open Access Nephrology Journals - A Guide for Nephrologists. Indian J Lib Inf Sci, 14 (2), 75-89.

21. Velmurugan, C. (2018). Nephrology Research Performance of Indian Scientists in Science Citation Index Expanded: A Scientometric Profile. JOJ uro \& nephron, 5 (2), 555658. DOI: 10.19080/JOJUN.2018.05.555658

22. Zhou X, Xing C, Xin L, Hu H, Li L, Fang J, Liu Z. (2012). Scientific publications in urology and nephrology journals from China: a 10-year analysis. Can Urol Assoc J, 6, $102-106$.

23. Exponential growth, accessed, https://en.wikipedia.org/wiki/Exponential_growth 